Quim. Nova, Vol. 34, No. 7, 1260-1264, 2011

\title{
ANÁLISE DE PESTICIDAS ORGANOCLORADOS EM ÁGUA USANDO A MICROEXTRAÇÃO EM FASE SÓLIDA POR HEADSPACE COM CROMATOGRAFIA GASOSA E ESPECTROMETRIA DE MASSAS
}

\author{
Crislaine Batista Prates, Sâmya Soler Gebara* e Nilva Ré-Poppi \\ Departamento de Química, Universidade Federal de Mato Grosso de Sul, Av. Senador Fillinto Mullher, 1555, Cidade Universitária, \\ 79080-190 Campo Grande - MS, Brasil
}

Recebido em 2/9/10; aceito em 16/2/11; publicado na web em 15/4/11

\begin{abstract}
ANALYSIS OF ORGANOCHLORINE PESTICIDES IN WATER USING HEADSPACE SOLID PHASE MICROEXTRACTION WITH GAS CHROMATOGRAPHY AND MASS SPECTROMETRY. A method based on headspace - solid phase microextraction coupled with gas chromatography - mass spectrometry was validated for the quantitative determination of 18 organochlorine pesticides in water. For the extraction conditioning some parameters as the best type of coating fiber, time and temperature of extraction, $\mathrm{pH}$ and ionic strength were evaluated. The method HS-SPME/GC-MS/MS showed linear coefficient above 0.9948 . The repeatability of the measurements were lower than 7.6\%. Relative recoveries were between 88 and $110 \%$. Limits of detection from $0.5 \times 10^{-3}$ to 1.0 $\mu \mathrm{g} \mathrm{L} \mathrm{L}^{-1}$ were obtained. A total of 31 samples were analyzed and 16 presented from 1 to 5 pesticides.
\end{abstract}

Keywords: organochlorine pesticides; HS-SPME/GC-MS/MS.

\section{INTRODUÇÃO}

O setor agrícola vem se esforçando a cada ano para aumentar a produção de alimentos, tanto para o mercado interno quanto para o externo. Porém, por falta de informação ou pelo interesse único no lucro, a produção agrícola contribuiu de forma efetiva para a contaminação das águas, tanto em nível superficial quanto subterrâneo. ${ }^{1}$

$\mathrm{O}$ estado de Mato Grosso do Sul (MS) em 2008 foi o $9^{\circ}$ estado em produção agrícola do país e, em função disto, o uso de pesticidas (defensivos agrícolas ou agrotóxicos) foi bastante intensificado. ${ }^{2}$ No distrito de Culturama, localizado na cidade de Fátima do Sul, as culturas temporárias (cana-de-açúcar, soja, mandioca, arroz, feijão e tomate) são predominantes e um grande número de inseticidas e acaricidas são aplicados. ${ }^{3} \mathrm{O}$ uso de pesticidas adquiridos de forma ilegal de países vizinhos (Paraguai e Uruguai) tem sido reportado pela imprensa brasileira, principalmente em alguns estados da Federação, como Rio Grande do Sul, Paraná e Mato Grosso do Sul, devido à proximidade com estes países. Os pesticidas podem ser adquiridos no Paraguai pagando-se até dez vezes menos o valor praticado no Brasil. ${ }^{4}$ Pelo fato destes produtos não passarem por um controle de qualidade, não se sabe exatamente a sua composição química e, consequentemente, a concentração que está sendo aplicada, elevando o risco à saúde humana e ao meio ambiente. O Ministério da Agricultura e a Polícia Federal têm intensificado ações para detectar, penalizar e coibir tais práticas.

Os organoclorados são compostos sintéticos de cadeia cíclica com baixa massa molecular e pouco solúveis em água e solúveis em solventes orgânicos, o que os torna mais tóxicos e de apreciável absorção cutânea. ${ }^{5}$ Além da via dérmica, são também absorvidos por via digestiva e respiratória. Devido à grande lipossubilidade e à lenta metabolização, esses compostos se acumulam na cadeia alimentar e no tecido adiposo. A eliminação se faz pela urina e também pelo leite materno. ${ }^{6}$ Por essas características, os pesticidas organoclorados têm sido progressivamente restringidos ou mesmo proibidos. As

*e-mail: samyasoler@hotmail.com primeiras restrições feitas ao seu uso foram ainda na década de $60 . \mathrm{O}$ hexaclorobenzeno (HCB) teve sua comercialização proibida em 1983 e neste ano a ANVISA (Agência Nacional de Vigilância Sanitária) divulgou que o endosulfan, por ser considerado altamente tóxico e associado a problemas do sistema endócrino, terá importação proibida a partir de julho de 2011, e a produção nacional encerrada em 2013. ${ }^{7}$

Os pesticidas clorados apresentam grande estabilidade química e pronunciada ação residual e devido a sua alta persistência no ambiente ainda são objetos de frequentes estudos. ${ }^{8-10}$

A cromatografia gasosa (GC; Gas Chromatography) com a utilização do detector por captura de elétrons (ECD; Electron Capture Detection) ou acoplado à espectrometria de massas (MS; Mass Spectrometry) é relatada como uma técnica bem definida para análise de organoclorados. ${ }^{11}$ A exatidão e a precisão na análise de pesticidas depende do preparo da amostra (extração e pré-concentração) e da performance do instrumento. A microextração em fase sólida (SPME, Solid Phase Microextraction) é uma técnica analítica capaz de integrar extração e concentração em uma única etapa e provou ser significativamente rápida, simples e de fácil manuseio, além de quimicamente limpa, pela não utilização de solventes. A extração no modo headspace por SPME (HS-SPME) tem sido utilizada para determinar pesticidas organoclorados em diferentes matrizes, como água, solo e fluidos biológicos, ${ }^{12,13}$ pois confere maior durabilidade à fibra por não entrar em contato com a amostra.

Neste contexto, o trabalho teve como objetivo desenvolver e validar um método alternativo para análise de pesticidas organoclorados usando HS-SPME/GC-MS/MS para análise de águas superficiais e subterrâneas.

\section{PARTE EXPERIMENTAL}

\section{Amostragem}

As amostras foram coletadas no distrito de Culturama, município de Fátima do Sul, Estado de Mato Grosso do Sul, nos meses de setembro e novembro de 2008. Foram coletadas 31 amostras de poços 
semiartesianos, em pequenas propriedades na zona rural deste distrito. A água dos poços é utilizada para abastecer as residências, no preparo dos insumos agrícola, na irrigação de culturas e no fornecimento para aviários e pocilgas. A profundidade dos poços amostrados varia entre 15 e $60 \mathrm{~m}$. As amostras foram coletadas na torneira que recebe água diretamente do poço, após se acionar a bomba por $5 \mathrm{~min}$, em frascos de vidro âmbar de 1 L (limpos conforme NBR 9898, ABNT, 1987). Os frascos foram acondicionados em caixa de isopor e mantidos sob refrigeração durante o transporte para o laboratório, onde foram conservados em geladeira $\left(4{ }^{\circ} \mathrm{C}\right)$ por um período máximo de 7 dias até as análises.

\section{Padrões e reagentes}

Os padrões dos pesticidas organoclorados foram adquiridos da Supelco (USA) em ampola de $1 \mathrm{~mL}$ (TLC Pesticides Mix, lote LB 30319), contendo $2000 \mu \mathrm{g} \mathrm{mL} \mathrm{mL}^{-1}$ de aldrin; $\alpha$-HCB; $\beta-\mathrm{HCB} ; \delta$-HCB; $\gamma$-HCB; dieldrin; endosulfan I (alfa); endosulfan II (beta); endosulfan sulfato; endrin; endrin cetona; endrin aldeído; heptacloro; heptacloro epóxido isômero B; metoxicloro; 4,4'-DDD; 4,4'-DDE e 4,4'-DDT. Foi utilizado pentacloronitrobenzeno com $99 \%$ de pureza, da Aldrich, como padrão interno. Foram preparadas soluções estoque contendo os 18 pesticidas organoclorados na concentração de $80 \mathrm{mg} \mathrm{L}^{-1}$ metanol (SupraSolv) da Merck (Darmstadt, Germany) e armazenadas a $-4{ }^{\circ} \mathrm{C}$. As soluções de trabalho foram preparadas em água ultrapura do sistema Milli-Q (Millipore da Bedford, MA, USA) e armazenadas a $4{ }^{\circ} \mathrm{C}$. Ácido acético e hidróxido de sódio usados no ajuste do $\mathrm{pH}$ foram da Merck, assim como o cloreto de sódio usado para teste de força iônica.

\section{Instrumentação}

As análises foram realizadas usando um cromatógrafo a gás GC-3900 acoplado a um espectrômetro de massas Saturn 2100 T/MS/MS da Varian, equipado com injetor modelo 1177 (splitsplitless) e analisador de massas do tipo íon trap. Aquisição de dados com software Saturn GC/MS 5.52 e biblioteca NIST 2.0. Para a separação cromatográfica foi empregada uma coluna capilar de sílica fundida com fase estacionária de polidimetilsiloxano com $5 \%$ de fenila (VF-5 ms, Factor Four) com $30 \mathrm{~m}$ de comprimento, $0,25 \mathrm{~mm}$ de d.i. e $0,25 \mu \mathrm{m}$ de espessura do filme. Hélio com teor de pureza de $99,999 \%$ foi utilizado como gás de arraste a um fluxo constante de $1,0 \mathrm{~mL} \mathrm{~min}^{-1}$. Temperatura do injetor de $250{ }^{\circ} \mathrm{C}$ e injeção splitless com tempo de amostragem de 2,0 min, seguido de uma razão de split de 50:1 por 15,0 min e de 20:1 no restante da corrida. As condições para o forno da coluna foram: temperatura inicial em $80{ }^{\circ} \mathrm{C}(4 \mathrm{~min}) ; 80-215^{\circ} \mathrm{C}\left(15^{\circ} \mathrm{C} \mathrm{min}{ }^{-1}\right.$, mantidos por $0,5 \mathrm{~min}) ; 215-230{ }^{\circ} \mathrm{C}\left(2{ }^{\circ} \mathrm{C} \mathrm{min}^{-1}\right.$, com $3 \mathrm{~min}$ de isotérmica); $230-260{ }^{\circ} \mathrm{C}\left(5^{\circ} \mathrm{C} \mathrm{min}{ }^{-1}\right.$, com 2 min de isotérmica). No detector fixou-se $200{ }^{\circ} \mathrm{C}$ na armadilha de íons, $50{ }^{\circ} \mathrm{C}$ no manifold e $250{ }^{\circ} \mathrm{C}$ na linha de transferência. A aquisição de íons no modo de análise em varredura linear (scan) foi efetuada no intervalo de 60-430 $\mathrm{m} / \mathrm{z}$. A ionização foi por impacto eletrônico com $70 \mathrm{eV}$, tempo de varredura de $0,5 \mathrm{~s} \mathrm{scan}^{-1}$ e 20000 de contagem de íons. A corrente de emissão do filamento de ionização foi de $10 \mu \mathrm{A}$ e a voltagem da eletromultiplicadora foi estabelecida na calibração automática.

\section{Análises por GC-MS/MS}

Após análise dos espectros de massas dos 18 pesticidas organoclorados selecionou-se um íon intenso de alta massa molecular para fragmentação com a energia estabelecida no estudo das amplitudes de excitação, no modo não ressonante (0-100 V). Os íons seleciona- dos para o desenvolvimento do método e as energias de excitação que geraram os íons de quantificação são mostrados na Tabela 1. A corrente no filamento foi de $10 \mu \mathrm{A}$, a voltagem na eletromultiplicadora foi estabelecida na calibração automática e ficou em torno de $1800 \mathrm{~V}$, a contagem de íons no trap foi $5000 \mathrm{com} 0,35 \mathrm{~s} \cdot \mathrm{scan}^{-1}$.

Tabela 1. Parâmetros de aquisição no modo de análise dos pesticidas

\begin{tabular}{lcccc}
\hline Pesticida & $\begin{array}{c}\text { Tempo de } \\
\text { retenção } \\
(\mathrm{min})\end{array}$ & $\begin{array}{c}\text { Íon } \\
\text { precursor } \\
(\mathrm{m} / z)\end{array}$ & $\begin{array}{c}\text { Íons } \\
\text { produtos } \\
(\mathrm{m} / \mathrm{z})\end{array}$ & $\begin{array}{c}\text { Amplitude da } \\
\text { energia de excita- } \\
\text { ção }(\mathrm{V})\end{array}$ \\
\hline$\alpha$-HCB & 13,208 & 183 & $109,146,148$ & 88 \\
$\gamma$-HCB & 13,606 & 219 & $109,146,148$ & 94 \\
PI & 13,670 & 295 & 237,265 & 92 \\
$\beta$-HCB & 13,764 & 183 & $109,146,148$ & 84 \\
$\delta$-HCB & 14,253 & 183 & $109,146,148$ & 78 \\
Heptacloro & 15,157 & 272 & 235,237 & 100 \\
Aldrin & 16,011 & 293 & $221,255,257$ & 99 \\
Heptacloro Epóxido & 17,055 & 353 & 263,317 & 100 \\
Endosulfan I & 18,217 & 339 & $266,301,337$ & 98 \\
4,4 DDE & 18,848 & 318 & 246,316 & 100 \\
Dieldrin & 19,162 & 277 & $204,239,241$ & 99 \\
Endrin & 19,972 & 281 & $243,245,279$ & 100 \\
Endosulfan II & 20,398 & 339 & $195,229,267$ & 98 \\
4,4 DDD & 20,516 & 235 & 165,199 & 95 \\
Endrin aldeído & 21,013 & 345 & $279,341,342$ & 99 \\
Endosulfan sulfato & 22,076 & 387 & $252,254,289$ & 95 \\
4,4DDT & 22,229 & 235 & 165,199 & 98 \\
Endrin cetona & 24,729 & 317 & $243,279,281$ & 100 \\
Metoxicloro & 25,491 & 227 & $141,153,281$ & 95 \\
\hline & & & &
\end{tabular}

\section{Procedimento de extração com SPME}

Foram avaliadas cinco fibras comerciais com diferentes recobrimentos: polidimetilsiloxano $100 \mu \mathrm{m}$ (PDMS), polidimetilsiloxano/divinilbenzeno $65 \mu \mathrm{m}$ (PDMS/DVB), divinilbenzeno/ carboxen/polidimetilsiloxano 50/30 $\mu \mathrm{m}$ (DVB/CAR/PDMS) e poliacrilato $85 \mu \mathrm{m}$ (PA), todas da marca Supelco (Bellefonte, PA, USA). Também foi avaliada a fibra NiTi-ZrO $\mathrm{rO}_{2}$ produzida na Universidade Federal de Santa Catarina pelo Prof. E. Carasek. ${ }^{14}$

Antes do uso, as fibras foram condicionadas conforme recomendado pelo fabricante. As extrações dos pesticidas foram efetuadas usando uma alíquota da amostra de $5 \mathrm{~mL}$ em vial de 15 $\mathrm{mL}$ com tampa fenólica, septo de PTFE/silicone de $11 \mathrm{~mm}$ e holder manual. Os diversos recobrimentos de fibra foram avaliados em extrações headspace em banho-maria sem agitação. Nesta etapa os experimentos foram realizados mantendo-se os tempos de extração e dessorção de 60 e 2 min, respectivamente, a temperatura de extração de $70{ }^{\circ} \mathrm{C}$ e pH da solução 6.

A fibra DVB/CAR/PDMS 50/30 $\mu$ m mostrou melhor resposta e menores coeficientes de variações para as triplicatas, sendo selecionada para outros experimentos.

Avaliou-se a seguir a extração headspace com agitação magnética e sem agitação em banho-maria. A extração headspace no modo estático apresentou maior eficiência. Usando a fibra DVB/ CAR/PDMS 50/30 $\mu \mathrm{m}$ no modo de extração headspace em banhomaria sem agitação estabeleceu-se o procedimento estudando-se 
os seguintes parâmetros: tempo de extração $(20,30,40,50,60$ e 70 min), tempo de dessorção $(60,90,120$ e $150 \mathrm{~s}), \mathrm{pH}$ (3, 6 e 9 ) e força iônica da solução $(10,20$ e $30 \%$ de $\mathrm{NaCl})$. Os estudos para determinar estes parâmetros foram realizados em solução contendo os 18 pesticidas organoclorados a uma concentração de $50 \mathrm{ng} \mathrm{L}^{-1}$. Todas as análises foram feitas em triplicata.

\section{Validação do método SPME-GC-MS/MS}

Os parâmetros analíticos investigados na validação do método foram seletividade, linearidade, sensibilidade (limites de detecção, LD e quantificação, LQ), precisão e exatidão. Para determinar a seletividade obteve-se o cromatograma da matriz, água ultrapura e água superficial não contaminada com organoclorado, e da matriz enriquecida com padrões (Figura 1).

Os limites de detecção e quantificação foram calculados baseados na razão sinal ruído dos picos, assumindo a razão 10:1 para LQ e 3:1 para LD. O método de quantificação foi o da padronização interna. As curvas analíticas foram obtidas para concentrações dos analitos entre $0,005-16 \mu \mathrm{g} \mathrm{L}^{-1}$. A precisão e a exatidão do método foram investigadas através de ensaios de recuperação relativa e coeficientes de variações das replicatas. Os testes de recuperação relativa foram feitos adicionando-se os analitos às amostras reais (água superficial) em três níveis de concentração para cada analito que foram estabelecidos em torno de 2,5 e 10 vezes o valor do limite de quantificação.

\section{Estudo de interferências}

Foram preparadas soluções contendo os 18 pesticidas organoclorados em soluções com 5, 10, 20, $40 \mathrm{mg} \mathrm{L}^{-1}$ de ácido húmico, em pH 6, com a finalidade de avaliar possíveis interferências da matéria orgânica na quantificação dos pesticidas em água superficiais. As concentrações avaliadas dos pesticidas foram $2,5 \mu \mathrm{g} \mathrm{L}^{-1}$ para $\delta$-HCB, endrin, endrin cetona, endosulfan II e metoxicloro, $8,5 \mu \mathrm{g}$ $\mathrm{L}^{-1}$ para endosulfan sulfato e para demais pesticidas a concentração foi $0,05 \mu \mathrm{g} \mathrm{\textrm {L } ^ { - 1 }}$. O ácido húmico utilizado nos experimentos foi extraído do solo da região da ilha de Cananeia, SP, com solução de $\mathrm{NaOH} 0,5 \mathrm{~mol} \mathrm{~L}^{-1}$ em atmosfera de $\mathrm{N}_{2}$ e purificado usando cromatografia líquida de troca iônica (IR - 120) até obtenção do teor de cinza inferior a $0,5 \%$.

\section{RESULTADOS E DISCUSSÃO}

\section{Otimização da técnica HS-SPME}

Os experimentos realizados para determinar o tempo de extração mostraram valores máximos para as áreas dos picos entre 50 e/ou 60 min de extração para 12 dos 18 pesticidas estudados, a temperatura de $70{ }^{\circ} \mathrm{C}$. Os pesticidas 4,4'-DDE, aldrin, heptacloro, 4,4'-DDD, dieldrin e 4,4'-DDT apresentaram áreas crescentes até $70 \mathrm{~min}$ de extração (maior valor estudado), não atingindo o equilíbrio. O tempo de $60 \mathrm{~min}$ foi selecionado para as análises subsequentes. Tempo e temperatura de dessorção foram estabelecidos, respectivamente, em 2 min e $260^{\circ} \mathrm{C}$. A fibra ficou inserida no injetor durante toda a corrida cromatográfica para limpeza (28 min) eliminando o "efeito de memória", caracterizado pelo dessorção incompleta dos analitos. Um branco da fibra foi obtido todos os dias antes do início das análises. $\mathrm{O}$ estudo da força iônica do meio mostrou que a extração feita sem adição $\mathrm{NaCl}$ obteve a melhor eficiência. Os resultados obtidos em $\mathrm{pH}$ ácido e básico foram inferiores a aquele em $\mathrm{pH}$ neutro.

\section{Validação do método}

Os cromatogramas obtidos das análises da água ultrapura e da amostra de água superficial (matriz isenta do analito) não apresentaram pico interferentes, conferindo seletividade ao método desenvolvido. A Tabela 2 mostra a faixa de linearidade investigada para os analitos ( $n=6)$, os coeficientes de correlação linear, LD, LQ e valores máximos estabelecidos pelo Ministério da Saúde na Portaria n. 518 de 2004. Foi observada linearidade com coeficiente de correlação linear (r) entre 0,9948 e 0,9985 . Os limites de detecção para o método permaneceram na faixa de $5,0 \times 10^{-4}$ a $1,0 \mu \mathrm{g} \mathrm{L}^{-1}$, e os limites de quantificação ficaram entre $3,3 \times 10^{-3}$ e $3,3 \mu \mathrm{g} \mathrm{L}^{-1}$, atendendo assim os valores estabelecidos pela legislação. Os valores de recuperação relativa situaram-se entre 88 e $110 \%$, com coeficientes de variações inferiores a 7,6\%, mostrados na Tabela 3 .

São relatados estudos prévios envolvendo a validação de metodologias para análise de pesticidas organoclorados em água usando a SPME-GC/MS. Alguns autores utilizaram analisador de massas do tipo íon trap no modo de análise em varredura linear (scan) e MS/MS. Outros utilizaram analisador de massas do tipo quadrupolo no modo de monitoramento de íon seletivo (SIM; Selective Ion Monitoring).

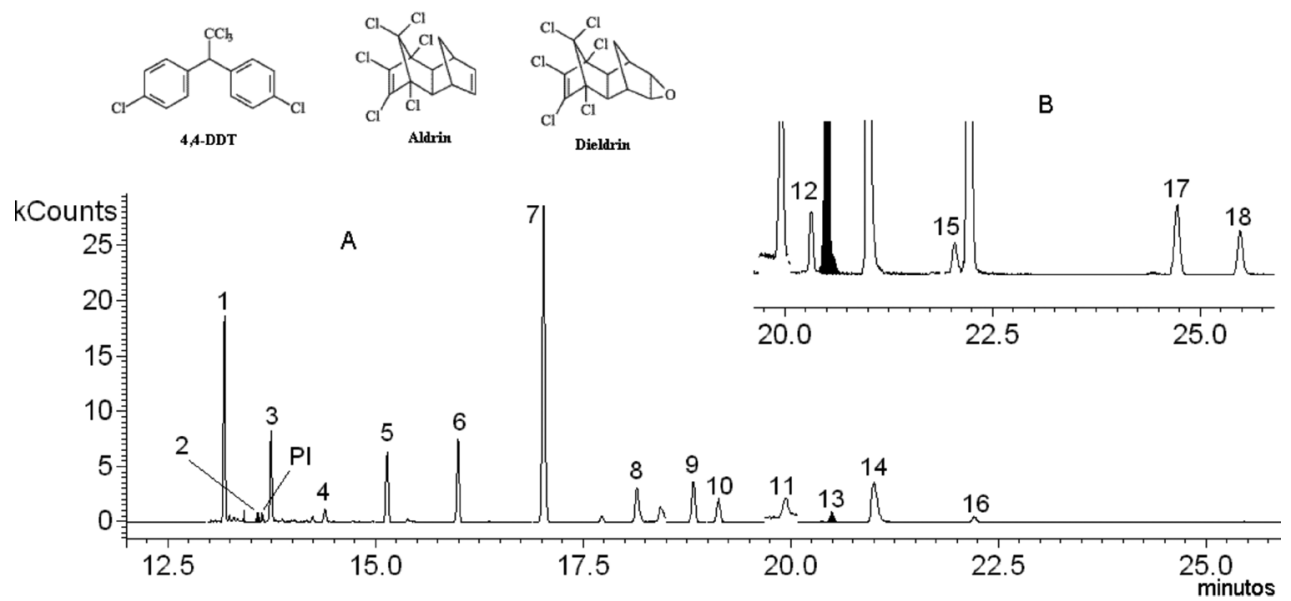

Figura 1. A: cromatograma obtido da análise por HS-SPME/GC-MS/MS de uma amostra fortificada com 0,5 $\mu g L^{-1}$ de pesticidas. B: ampliação do cromatograma (20-26 min) da amostra fortificada com 8,5 $\mu \mathrm{g} \mathrm{L}^{-1}$. Picos: 1: $\alpha-H C B, 2: \gamma-H C B$, PI: padrão interno pentacloronitrobenzeno, 3: $\beta$-HCB, 4: $\delta H C B, 5$ : heptacloro, 6: aldrin, 7: heptacloro epóxido, 8: endosulfan I, 9: 4,4'-DDE, 10: dieldrin, 11: endrin, 12: endossulfan II, 13: 4,4'-DDD, 14: endrin aldeído, 15: endossulfan sulfato, 16: 4,4'-DDT, 17: endrin cetona, 18: metoxicloro 
Tabela 2. Parâmetros analíticos obtidos para o método HS-SPME/GC-MS/MS

\begin{tabular}{|c|c|c|c|c|c|}
\hline Pesticidas & $\begin{array}{l}\text { Faixa Linear } \\
\quad\left(\mu \mathrm{g} \mathrm{L}^{-1}\right)\end{array}$ & $\mathrm{r}$ & $\begin{array}{c}\mathrm{LD} \\
\left(\mu \mathrm{g} \mathrm{L}^{-1}\right)\end{array}$ & $\begin{array}{c}\mathrm{LQ} \\
\left(\mu \mathrm{g} \mathrm{L} \mathrm{L}^{-1}\right)\end{array}$ & $\begin{array}{c}\text { Portaria } \mathrm{MS} \mathrm{n}^{\circ} 518,2004 \\
\mu \mathrm{g} \mathrm{L}^{-1}\end{array}$ \\
\hline$\alpha-\mathrm{HCB}$ & $0,05-0,5$ & 0,9985 & $1,0 \times 10^{-2}$ & $3,3 \times 10^{-2}$ & 1 \\
\hline$\gamma-\mathrm{HCB}$ & $0,05-0,5$ & 0,9966 & $1,0 \times 10^{-2}$ & $3,3 \times 10^{-2}$ & 2 \\
\hline$\beta-\mathrm{HCB}$ & $0,05-0,5$ & 0,9965 & $1,0 \times 10^{-2}$ & $3,3 \times 10^{-2}$ & 1 \\
\hline$\delta$-HCB & $0,5-2,5$ & 0,9972 & $5,0 \times 10^{-2}$ & $1,7 \times 10^{-1}$ & 1 \\
\hline Heptacloro & $0,05-0,5$ & 0,9981 & $5,0 \times 10^{-3}$ & $1,6 \times 10^{-2}$ & $3 \times 10^{-2}$ \\
\hline Aldrin & $0,005-0,05$ & 0,9976 & $5,0 \times 10^{-4}$ & $1,5 \times 10^{-3}$ & $3 \times 10^{-2}$ \\
\hline Heptacloro epóxido & $0,005-0,05$ & 0,9982 & $5,0 \times 10^{-4}$ & $1,5 \times 10^{-3}$ & $3 \times 10^{-2}$ \\
\hline Endosulfan I & $0,005-0,05$ & 0,9978 & $1,0 \times 10^{-3}$ & $3,0 \times 10^{-3}$ & 20 \\
\hline 4,4'- DDE & $0,005-0,05$ & 0,9948 & $5,0 \times 10^{-4}$ & $1,5 \times 10^{-3}$ & 2 \\
\hline Dieldrin & $0,005-0,05$ & 0,9959 & $1,0 \times 10^{-3}$ & $3,0 \times 10^{-3}$ & $3 \times 10^{-2}$ \\
\hline Endrin & $0,5-2,5$ & 0,9982 & $5,0 \times 10^{-2}$ & $1,7 \times 10^{-1}$ & $6 \times 10^{-1}$ \\
\hline Endosulfan II & $1,7-8,5$ & 0,9974 & $5,0 \times 10^{-1}$ & 1,7 & 20 \\
\hline $4,4^{\prime}-\mathrm{DDD}$ & $0,005-0,05$ & 0,9951 & $1,0 \times 10^{-3}$ & $3,0 \times 10^{-3}$ & 2 \\
\hline Endrin aldeído & $0,5-2,5$ & 0,9966 & $5,0 \times 10^{-2}$ & $1,7 \times 10^{-1}$ & \\
\hline Endosulfan sulfato & $3,3-16,0$ & 0,9952 & 1,0 & 3,3 & - \\
\hline $4,4^{\prime}-\mathrm{DDT}$ & $0,005-0,05$ & 0,9971 & $1,0 \times 10^{-3}$ & $3,0 \times 10^{-3}$ & 2 \\
\hline Endrin cetona & $0,5-2,5$ & 0,9978 & $5,0 \times 10^{-2}$ & $1,7 \times 10^{-1}$ & - \\
\hline Metoxicloro & $1,7-8,5$ & 0,9982 & $5,0 \times 10^{-1}$ & 1,7 & 20 \\
\hline
\end{tabular}

$\mathrm{r}=$ coeficiente de correlação linear; $\mathrm{LD}=$ limite de detecção; LQ=limite de quantificação; - valores não estabelecidos na Portaria ${ }^{\circ}$ 518/04.

Tabela 3. Recuperação relativa e precisão do método

\begin{tabular}{|c|c|c|c|c|c|c|c|c|c|c|c|c|c|c|c|c|c|c|c|c|}
\hline \multirow[b]{2}{*}{ Pesticida } & \multicolumn{2}{|c|}{$0,005 \mu \mathrm{g} \mathrm{L}{ }^{-1}$} & \multicolumn{2}{|c|}{$0,01 \mu \mathrm{g} \mathrm{L} \mathrm{L}^{-1}$} & \multicolumn{2}{|c|}{$0,05 \mu \mathrm{g} \mathrm{\textrm {L } ^ { - 1 }}$} & \multicolumn{2}{|c|}{$0,1 \mu \mathrm{g} \mathrm{L}^{-1}$} & \multicolumn{2}{|c|}{$0,5 \mu \mathrm{g} \mathrm{L}^{-1}$} & \multicolumn{2}{|c|}{$1,7 \mu \mathrm{g} \mathrm{L}^{-1}$} & \multicolumn{2}{|c|}{$2,5 \mu \mathrm{g} \mathrm{L}-1$} & \multicolumn{2}{|c|}{$5,0 \mu \mathrm{g} \mathrm{L}^{-1}$} & \multicolumn{2}{|c|}{$8,5 \mu \mathrm{g} \mathrm{L}^{-1}$} & \multicolumn{2}{|c|}{$16 \mu \mathrm{g} \mathrm{L}^{-1}$} \\
\hline & $\mathrm{R}$ & $\mathrm{CV}$ & $\mathrm{R}$ & $\mathrm{CV}$ & $\mathrm{R}$ & $\mathrm{CV}$ & $\mathrm{R}$ & $\mathrm{CV}$ & $\mathrm{R}$ & $\mathrm{CV}$ & $\mathrm{R}$ & $\mathrm{CV}$ & $\mathrm{R}$ & $\mathrm{CV}$ & $\mathrm{R}$ & $\mathrm{CV}$ & $\mathrm{R}$ & $\mathrm{CV}$ & $\mathrm{R}$ & $\mathrm{CV}$ \\
\hline & & & & & & & & & $(\%)$ & & & & & & & & & & & \\
\hline Aldrin & 102,9 & 5,0 & 98,1 & 2,6 & 101,9 & 4,4 & & & & & & & & & & & & & & \\
\hline Heptacloro epóxido & 93,8 & 2,0 & 95,1 & 0,7 & 96,7 & 2,0 & & & & & & & & & & & & & & \\
\hline Endosulfan I & 101,6 & 4,5 & 99,2 & 2,5 & 98,3 & 3,0 & & & & & & & & & & & & & & \\
\hline 4,4'-DDE & 98,2 & 6,1 & 100,9 & 4,4 & 97,6 & 2,0 & & & & & & & & & & & & & & \\
\hline Dieldrin & 98,6 & 2,6 & 103,1 & 6,0 & 99,4 & 3,9 & & & & & & & & & & & & & & \\
\hline 4,4'-DDD & 96,8 & 5,7 & 99,8 & 5,7 & 99,8 & 3,0 & & & & & & & & & & & & & & \\
\hline 4,4'-DDT & 95,2 & 1,2 & 98,3 & 0,9 & 100,8 & 1,6 & & & & & & & & & & & & & & \\
\hline$\alpha-\mathrm{HCB}$ & & & & & 101,9 & 6,4 & 100,1 & 3,8 & 100,2 & 3,1 & & & & & & & & & & \\
\hline$\gamma-\mathrm{HCB}$ & & & & & 100,2 & 4,4 & 106,3 & 6,1 & 106,2 & 6,2 & & & & & & & & & & \\
\hline$\beta-\mathrm{HCB}$ & & & & & 97,2 & 6,7 & 98,8 & 5,7 & 99,0 & 3,0 & & & & & & & & & & \\
\hline Heptacloro & & & & & 106,3 & 5,9 & 96,6 & 1,9 & 101,1 & 5,7 & & & & & & & & & & \\
\hline$\delta$-HCB & & & & & & & & & 100,3 & 2,7 & 100,0 & 3,7 & 102,2 & 3,3 & & & & & & \\
\hline Endrin & & & & & & & & & 93,8 & 4,9 & 93,1 & 6,9 & 95,4 & 7,6 & & & & & & \\
\hline Endrin cetona & & & & & & & & & 102,5 & 6,1 & 99,4 & 3,6 & 99,0 & 2,6 & & & & & & \\
\hline Endrin aldeído & & & & & & & & & 105,4 & 4,6 & 109,7 & 1,6 & 103,4 & 1,8 & & & & & & \\
\hline Endosulfan II & & & & & & & & & & & & & 104,9 & 3,2 & 101,9 & 1,5 & 101,2 & 2,7 & & \\
\hline Metoxicloro & & & & & & & & & & & & & 91,6 & 2,6 & 103,9 & 4,9 & 97,6 & 2,6 & & \\
\hline Endosulfan sulfato & & & & & & & & & & & & & & & 90,8 & 5,2 & 95,8 & 2,9 & 87,9 & 3,8 \\
\hline
\end{tabular}

* R: Recuperação relativa; CV: Coeficiente de variação 
Ambos obtiveram valores de limites de detecção entre $6,0 \times 10^{-3} \mu \mathrm{g} \mathrm{L}^{-1}$ e $10 \mu \mathrm{g} \mathrm{L}{ }^{-1}$, mostrando que os resultados encontrados neste trabalho são da mesma ordem de grandeza daqueles obtidos com diferentes analisadores e/ou modo de análise (scan, SIM e MS/MS). ${ }^{15,16}$

\section{Estudo de interferentes}

O método SPME tem sido aplicado para encontrar a concentração livre de poluentes em soluções aquosas de ácido húmico e determinar os coeficientes de partição dos analitos. ${ }^{17}$

Neste trabalho, os valores porcentuais encontrados de recuperações dos analitos, em presença de ácido húmico, situaram-se entre 88 e $110 \%$, com coeficiente de variação entre 0,7 e 7,6\%. Estes resultados sugerem que em $\mathrm{pH}$ 6, temperatura de $70{ }^{\circ} \mathrm{C}$ e baixas concentrações dos pesticidas, condições em que os experimentos foram realizados, não ocorre adsorção dos pesticidas no ácido húmico e/ou a dessorção é favorecida. Não havendo interferência o método pode ser aplicado na determinação dos pesticidas em amostras de águas superficiais.

\section{Análises de amostras reais}

Os resultados das análises dos pesticidas organoclorados nas amostras de águas coletadas em Culturama mostraram a presença destes analitos em 16 amostras das 31 analisadas. Dos 18 pesticidas organoclorados estudados 7 foram detectados e 4 quantificados. Os valores de concentração dos pesticidas nas amostras variaram entre 1,9-153 ng L-1. O 4,4'-DDT não foi detectado nas amostras, porém seus metabólitos, o 4,4'-DDD e o 4,4'-DDE sim. O 4,4'-DDD foi quantificado em uma amostra com $3,8 \mathrm{ng} \mathrm{L}^{-1}$. O 4,4'-DDE foi encontrado em 12 amostras e quantificado em 7 delas com concentrações entre 1,9 e 9,0 ng L-1 , comprovando que é o metabólito mais estável. ${ }^{18}$

O heptacloro sofre epoxidação tornando-se o heptacloro epóxido, pertencentes à classe toxicológica II, altamente tóxicos, apresentando uma longa persistência no ambiente. ${ }^{19} \mathrm{O}$ heptacloro epóxido, a exemplo de seu precursor, apresentou valores inferiores $\left(2,87 \mathrm{ng} \mathrm{L}^{-1}\right)$ ao valor máximo permitido estipulado pela atual Portaria que é de 30 ng L $\mathrm{L}^{-1}$. O endrin é um inseticida pertencente à classe toxicológica I, usado principalmente em culturas de cana-de-açúcar, soja, algodão e milho. Este possui efeitos tóxicos similares ao aldrin e dieldrin, mas é menos estável. ${ }^{20} \mathrm{O}$ valor de $153 \mathrm{ng} \mathrm{L}^{-1}$ para endrin cetona foi encontrado em uma das amostras. Esta substância não está entre os pesticidas relacionados pela Portaria $n^{\circ} 518 / 04$, que estabelece valor máximo de $600 \mathrm{ng} \mathrm{L}^{-1}$ apenas para o endrin.

\section{CONCLUSÃO}

O método HS-SPME/GC-MS/MS desenvolvido neste trabalho para determinação de 18 pesticidas organoclorados em água apresentou boa seletividade, linearidade, sensibilidade, precisão e exatidão adequadas e foi aplicado com sucesso nas análises de amostras reais. Os limites encontrados foram baixos suficientes para se detectar essas substâncias a um nível inferior ao estabelecido pela legislação nacional.
A presença de matéria orgânica, mesmo em diferentes concentrações, não interferiu nas extrações, atribuindo ao método robustez e aplicabilidade a amostras sujas como de águas superficiais.

Nas amostras de águas dos poços de monitoramento do distrito de Culturama foram detectados 7 pesticidas organoclorados, sendo o endrin cetona o mais abundante. Devido aos efeitos que estes pesticidas podem causar à saúde e ao meio ambiente o monitoramento das águas é importante para a qualidade de vida.

\section{AGRADECIMENTOS}

Ao apoio financeiro fornecido pela FUNDECT, CAPES, CNPq e PROPP-UFMS.

\section{REFERÊNCIAS}

1. Shukla, G.; Kumar, A.; Bhanti, M.; Joseph, P. E.; Taneja, A.; Environ. Int. 2006, 32, 244.

2. Tsunechiro, A.; Coelho, P. J.; Miura, M.; Análise e Indicadores do Agronegócio 2010, 5, 3.

3. http://www.ibge.gov.br/cidadesat/link.php?uf=ms, acessada em Dezembro 2010.

4. h t t p : / / fantastico.globo.com/Jornalis mo/ FANT/0,,MUL1509636-15605,00.html, acessada em Dezembro 2010.

5. Hermes, L. C.; Silva, A. S.; Avaliação da qualidade das águas: Manual Prático, Embrapa Informação Tecnológica: Brasília, DF, 2004.

6. OPAS (Organização Pan-Americana da Saúde); Manual de Vigilância da Saúde de Populações Expostas a Agrotóxicos, Brasília, 1997.

7. http://www.revistacafeicultura.com.br, acessada em Agosto 2010.

8. Rissato, S. R.; Libânio, M.; Giafferis, G. P.; Gerenutti, M.; Quim. Nova 2004, 27, 739 .

9. Arruda, T. L.; Jardim, W. F.; Quim. Nova 2007, 30, 1628.

10. Menezes, A. F.; Santos, F. N.; Pereira, P. A. P.; Microchem. J. 2010, 96, 139.

11. Zacharis, C. K.; Tzanavaras, P. D.; Roubos, K.; Dhima, K.; J. Chromatogr., A 2010, 1217, 5896.

12. Ratola, N.; Santos, L.; Herbert, P.; Alves, A.; Anal. Chim. Acta 2006, 573, 202.

13. Derouiche, A.; Driss, M. R.; Morizur, J. P.; Taphanel, M. H.; J. Chromatogr., A 2007, 1138, 231.

14. Carasek, E.; Martendal, E.; Budziak, D.; Anal. Chim. Acta 2007, 598, 254.

15. Komatsu, E.; Vaz, J. M.; Quim. Nova 2004, 27, 720.

16. Sakamoto, M.; Tsutsumi, T.; J. Chromatogr., A 2004, 1028, 63.

17. Prosen, H.; Fingler, S.; Zupancic-Kralj, L.; Drevenkar, V.; Chemosphere 2007, 66, 1580.

18. D’amato, C.; Torres, J. P. M.; Malm, O.; Quim. Nova 2002, 25, 995.

19. Calheiros, D. F.; Dissertação de Mestrado, Universidade de São Paulo, Brasil, 1993.

20. Rodriguez, M. P.; Tese de Doutorado, Universidade de São Paulo, Brasil, 2001. 